\title{
AGGREGATION AND DISAGGREGATION IN DEMAND FORECASTING
}

\author{
Paul Giesberts, Eindhoven University of Technology, NL \\ Jacob Wijngaard, University of Groningen, NL
}

An important element in production planning and control, especially in make-to-stock and assemble-to-order situations, is demand forecasting. Two, related, questions with respect to aggregation and disaggregation are important here. The first question is how to forecast an aggregate (capacity) variable. It is interesting to compare here direct aggregate forecasting and aggregation of detailed forecasts. The second question is how to forecast detailed (item) variables. In this case it is interesting to compare direct detailed forecasting and disaggregation of aggregate forecasts.

There are two important performance elements in forecasting. The first is that the forecast reacts well on a systematic change, the second is that it does not overreact on disturbances. The following simple (auto regressive) time series model appears to be useful in getting insight in these two sides of the performance of direct aggregate forecasting versus aggregating item forecasts.

$$
\begin{aligned}
& x(t)=x(t-1)+\in(t) \\
& d(t)=x(t)+\eta(t)
\end{aligned}
$$

With $x(),. d(),. \in(),. \eta($.$) vectors and \epsilon_{1}(t)$ and $\eta_{1}(t)$ normally distributed.

To compare direct item forecasts with disaggregating aggregate forecasts it is more appropriate to use a two phase demand model: In the first phase total demand is determined, in the second phase the distribution over the various categories is determined.

The results give insight in the strengths and weaknesses of aggregate versus detailed forecasting. 